\title{
NUMERICAL STUDY OF STALL INCEPTION IN A TRANSONIC AXIAL COMPRESSOR ROTOR BASED ON THE THROTTLE MODEL
}

\author{
Xiaocheng Zhu, Bo Liu, Jiangfeng Hu, Xin Shen \\ School of Mechanical Engineering, Shanghai Jiao Tong University, Shanghai, China \\ e-mail:zhxc@sjtu.edu.cn; liubo@sjtu.edu.cn; jiangfenghu1981@163.com; shenxin@sjtu.edu.cn
}

\begin{abstract}
The goal of the current paper is to investigate inner flow behavior on stall inception in a transonic compressor rotor. The stall inception process is numerically carried out by unsteady 3-D simulations based on the throttle model. The current study shows that stall starts from the tip of the blade, and stall cell extends to the axial, circumferential and radial directions. Through the comparison of flow transition characteristics at different flow rate conditions, the interface between the incoming flow and tip clearance flow shifts forward to the upstream as the mass flow decreases. Eventually, the shock detaches from the blade leading edge, and tip clearance flow spills into the adjacent blade passage, thus stall happens in the affected blade passages.
\end{abstract}

Keywords: stall, axial compressor, throttle, tip clearance flow

\section{Introduction}

The instability phenomena, rotating stall and surge in compressors limit the operating range of gas turbine engines. Rotating stall can cause fatal damages to compressors, which should be avoided. Many experimental and numerical programs which focus on the physical process of stall inception have been reported in order to avoid instabilities and to extend the operating range.

The mechanism of stall inception has become a research point since the 1950s. Three representative theories were established due to active research efforts. Emmons et al. (1955) considered that choke in some blade passages can induce an increase of incidences surrounding them. These large incidences change may result in stall cells that rotate in the opposite direction from the rotor. The second is the famous M-G model developed by Moore and Greitzer (1986). They thought that stall was induced by representative disturbance which was named the rotating wave. A long-length disturbances (modal) theory was developed. Day (1993) found a second type of the stall inception mechanism, short-length, or spike, disturbances. Those were disturbances with a length scale of the order of blade passage range compared to the length of the order of the rotor circumference of the inception model. The common view of two length scale inceptions was that stall was caused by rotation disturbances. Vo et al. (2008) described the last representative standpoint that was recognized as a criterion for spike initiated rotating stall inception. His comprehensive study indicated that leading edge tip clearance flow spillage and backflow at the trailing edge were two criteria for rotating stall inception. That theory was demonstrated by Hah et al. (2006) in a transonic axial rotor.

Recently, the mechanism of stall inception has been discussed in two main aspects, the tip clearance flow characteristic and the stall developmental process, see Hah et al. (2006), He (1997), Hoying et al. (1999), Hah et al. (1999, 2004), Furukawa et al. (2000), Yamada et al. (2007). It is well known that the experiment is the most straightforward way for researching. However, experimental measurement is difficult or even impossible to examine the detail flow structure in blade rows. In that case, numerical simulation can be an alternative and complementary way to 
experimental measurement in analysis of complex flow phenomena. During the past 15 years, Davis and Yao (2006), Chen et al. (2008), Yao et al. (2010) achieved some valuable results of the flow structure leading to stall by using computational fluid dynamics (CFD) methods.

At present, there are two primary CFD methods to simulate stall development in turbine blades. One is the plenum chamber model applied by Niazi (2000), the other is the throttle model commended by Cumpsty (1989). The throttle model is developed to compute the stall process of transonic axial compressor rotor-Rotor 37. Firstly, the stall inception process is simulated by using this throttle model, and then the numerical results from different conditions are examined in detail to analyze the relevant flow mechanism of stall.

The current study is based on numerical simulations of NASA Rotor 37 with the throttle model. The throttle coefficient is adjusted to control mass flow rates, which will lead the rotor flow from stable conditions to stall. The results are validated by experiment data. Then CFD results from stable conditions to the near stall condition are used to illustrate the stall developmental process. The mechanism of stall inception is concluded in the present paper.

\section{Numerical model and method}

\section{1. $\quad$ Numerical model}

NASA Rotor 37 is investigated in the present work. It is a transonic axial compressor rotor, originally designed and tested at NASA Lewis Research Center in the 1970s. The rotor has 36 blades with an aspect ratio of 1.19 and a hub-tip ratio of 0.7 . The inlet Mach number is 1.48 at the tip under the design speed of $17188.7 \mathrm{rpm}$. The design mass flow is $20.19 \mathrm{~kg} / \mathrm{s}$ and the pressure ratio is 2.106 . The choke mass flow is about $20.93 \mathrm{~kg} / \mathrm{s}$. The running tip clearance was estimated to be $0.0356 \mathrm{~cm}$ for the blind test case using both touch probe and rub probe measurements.

\subsection{Numerical method}

The unsteady simulations are carried out to investigate the rotor passage inner flow, and also to obtain instantaneous flow structures during stall inception by solving unsteady three-dimensional, Reynolds-averaged Navier-Stokes equations, through commercial solver package ANSYS CFX. This solver is based on the finite volume scheme, and utilizes second-order high precision discretization scheme. The $\kappa-\varepsilon$ turbulence model with the standard wall function is used to account for the turbulence flow. The simulation result was validated with the experimental data by $\mathrm{Hu}$ et al. (2013). 20 physical time steps are calculated for a single blade passage, and 10 inner iterations are performed at each time step for time accurate simulation in the present work. The total pressure, total temperature and flow angles are fixed as the inlet conditions. Non-slip and adiabatic conditions are imposed on the solid walls. The outlet boundary conditions are given in two types. For normal simulations, the static pressure is given with a simple radial equilibrium equation at the outlet. During stall conditions, the compressor pressure rise drops and the traditional exit static pressure cannot match the pressure drop. In that case, the current research applies the throttle model, which allows variation of the exit static pressure to match the compressor exit mass corrected to the exit total condition. The formula is as follows

$$
P_{\text {out }}=P_{\text {ambient }}+k k_{b} \frac{\rho}{2}\left(\frac{C_{x e}}{U}\right)^{2}
$$

where $\rho$ is density, $C_{x e}$ is velocity in the axial direction, $U$ is the middle span blade speed, $k_{b}$ is the reference coefficient, $k$ - throttle coefficient and $P_{\text {out }}$ is the pressure at the exit.

The grid is constructed by CFX-Turbo grid as shown in Fig. 1a. The computational grid for a single blade passage consists of 106 nodes in the streamwise direction, 43 nodes in the pitchwise 
direction, and 74 nodes in the spanwise direction. To capture the tip leakage flow correctly, H-type grid are embedded in the region of the tip clearance, which has $59 \times 16 \times 24$ nodes in the chordwise, spanwise and pitchwise directions, respectively. The monitor points at the tip area of blades are shown in Fig. 1b. However, limited by computation resources, six blade passages are built for unsteady simulation in order to capture the initial motion of the stall cell. Unsteady single-passage simulations of a single blade-row rotor were also performed by Vo et al. (2008) and Davis and Yao (2006) to capture the stall inception. Judging from Figs. 7 and 8, six flow passages are fit to simulate the initial development of the stall cell.

(a)

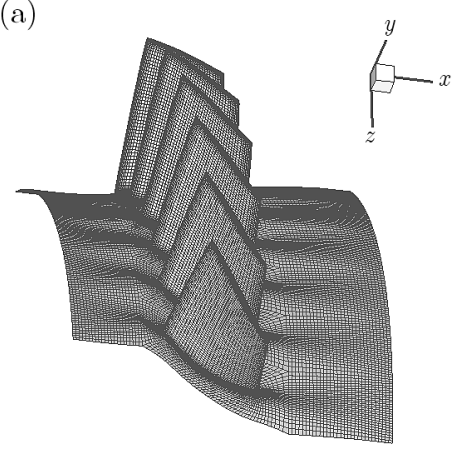

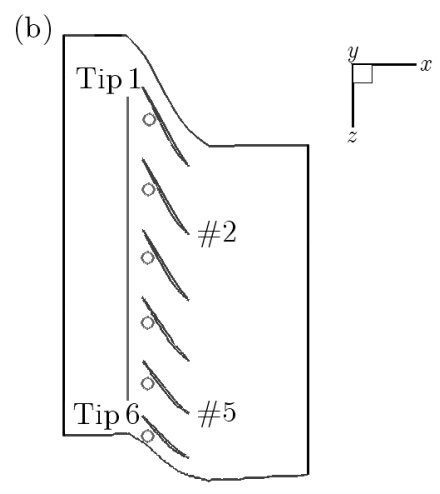

Fig. 1. (a) The computational grids with six passages. (b) The monitors at blade tip area

\section{Results and discussion}

\subsection{Validity of the numerical method}

The simulations are conducted with two kinds of outlet boundaries, pressure outlet and throttle model, respectively. The comparison between the numerical and experimental total pressure ratio characteristics is depicted in Fig. 2. The corrected mass flow rates are normalized by the value at choke. Although the predictions of simulation with boundary conditions of the pressure outlet are somewhat less than the experimental data, the trend of the total pressure ratio agrees very well with the experimental results. The predicted mass flow ratio at the near stall condition is slightly smaller than in the experimental data. Generally, the total pressure ratio characteristics are very close in the common mass flow area by those two methods.

In Fig. 2, we can also find the total pressure ratio drops from the peak at $k=0.57$. Figure 3 is a monitor of the history of pressure at the blade tip area for this condition. The static pressures are also very close at every passage versus time and have no significant phasic variety. It indicates that the compressor is still stable at this condition although the total pressure ratio decreases.

\subsection{Development of the rotating stall}

In order to simulate the stall process, the throttle coefficient is adjusted to $k=0.60$, which means that the mass flow rate decreases. Figure 4 shows the time histories of total pressure ratio, outlet static pressure, inlet mass flow and outlet mass flow. All these four monitored quantities sharply decline after nine revolutions of the rotor. This phenomenon indicates that the compressor comes into stall gradually. Four the time instants $T_{1}, T_{2}, T_{3}$ and $T_{4}$ are signed to analyze the flow change in stall inception process.

Figure 5 shows the static pressure coefficient distribution at the blade tip area in 4 time instants. At time $T_{1}$, the distribution of pressure is consistent between blades \#2 and \#5. They are all in the normal pressure distribution character. At $T_{2}$, some difference exists in the front part of the cascade. The flow periodical characteristic at passages breaks down at this time 


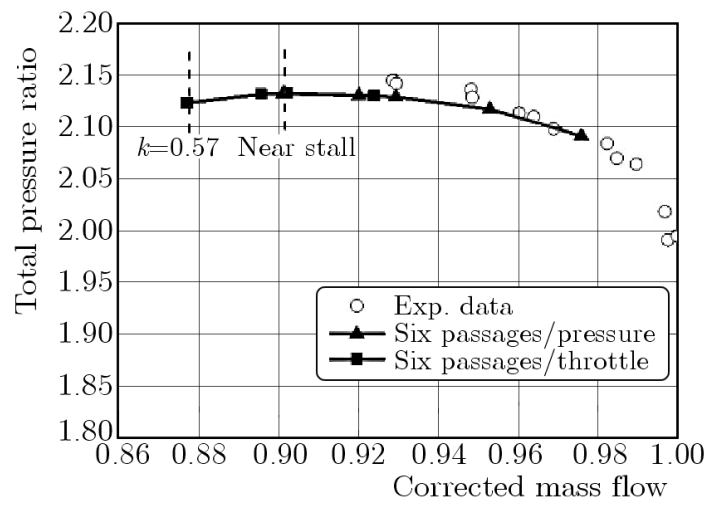

Fig. 2. The pressure characteristic curve of the compressor rotor

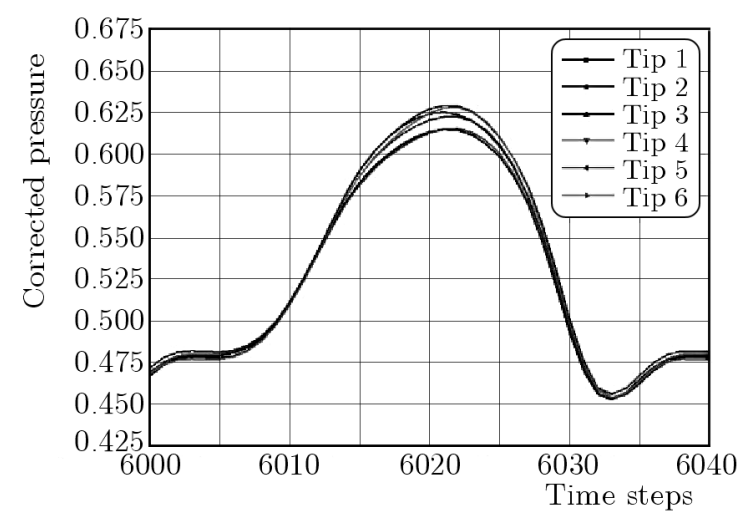

Fig. 3. The history of pressure at the blade tip
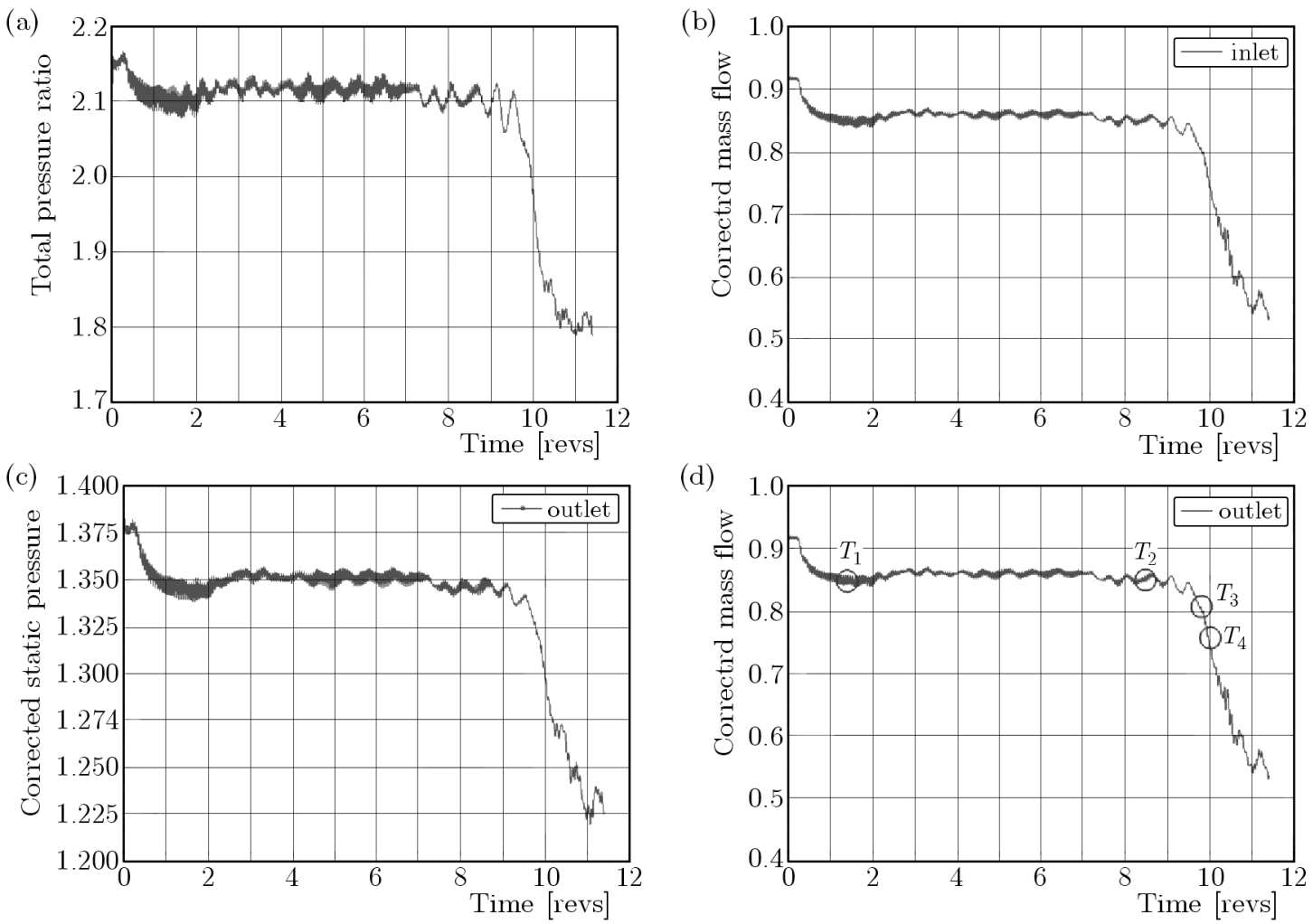

Fig. 4. Total pressure ratio, outlet static pressure, inlet mass and outlet mass curves versus time. The time is expressed in terms of subsequent revolutions of the rotor (from 0 up to 12 full turns) during numerical simulation 
to a certain extent. A nonaxisymmetric flow begins to take place in the passages. At $T_{3}$, the pressure coefficient distributions are entirely different along the blade chord, especially on the suction surface. The above analysis shows that the flow deteriorates more and more severely as the revolution number increases.

Stall is considered to be associated with the reverse flow (negative axial flow). Thus, the current paper now will examine the stalling process more deeply. The axial negative velocity distributions on surfaces at the blade tip and 1/3 axial chord cut plane before the leading edge of revolution are shown at four time instants, which represents the inner flow transformation from stable condition to stall.
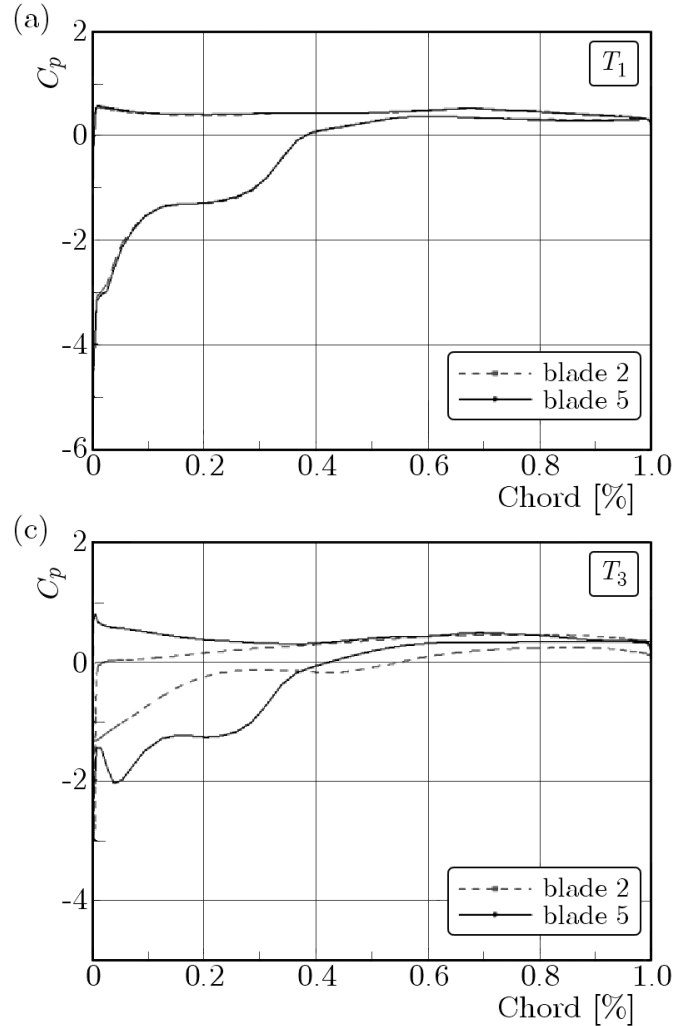
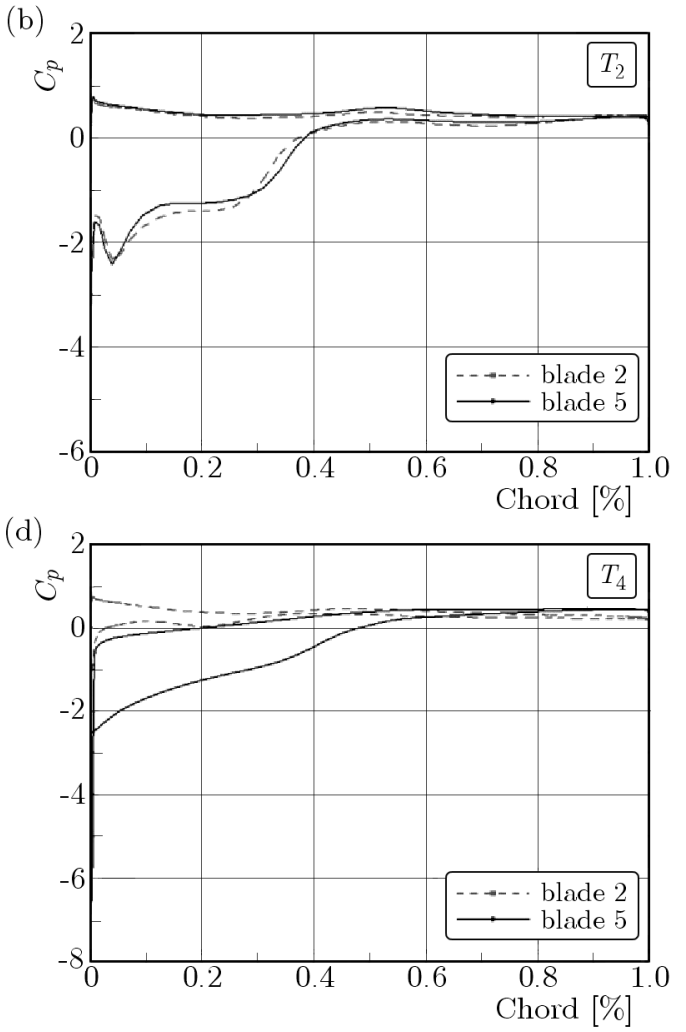

Fig. 5. The static pressure coefficient distribution at the blade tip in 4 time instants

In Fig. 6, we can see contours of the axial velocity which have been truncated so that only the negative velocity has a color. All positive velocities are colored white. At $T_{1}$, the distributions of axial negative velocity are almost uniform in six passages. At $T_{2}$, the flow asymmetry appears, and some passages appear a larger blockage area near the tip compared with others. At $T_{3}$, the large reverse flow area shows up among two blades passages, which is considered as the original form of the stall cell. At $T_{4}$, the reversed flow area develops bigger than $T_{3}$ and changes its position to the adjacent blade passages. This phenomenon indicates that the stall cell grows up with expanding in the axial and circumferential directions, and keeps occupying a larger area. Figure 7 shows the axial negative velocity distribution at $1 / 3$ of the axial chord cut plane in 4 time instants. As can bee seen in these figures, the reverse flow moves forward at $1 / 3$ of the axial chord plane at $T_{3}$, develops and expands rapidly along the radial and circumferential directions at $T_{4}$. The calculated rotating stall cell roughly covers two blade passages in the circumferential direction and about 10 to $20 \%$ span from the tip.

The above flow analysis describes the stall inception and developmental process. It can be concluded that the stall starts from the blade tip. At first, only one stall cell appears. Then, it expands in the axial, circumferential and radial directions, while rotates with a lower speed than the blade in the same rotating direction. 

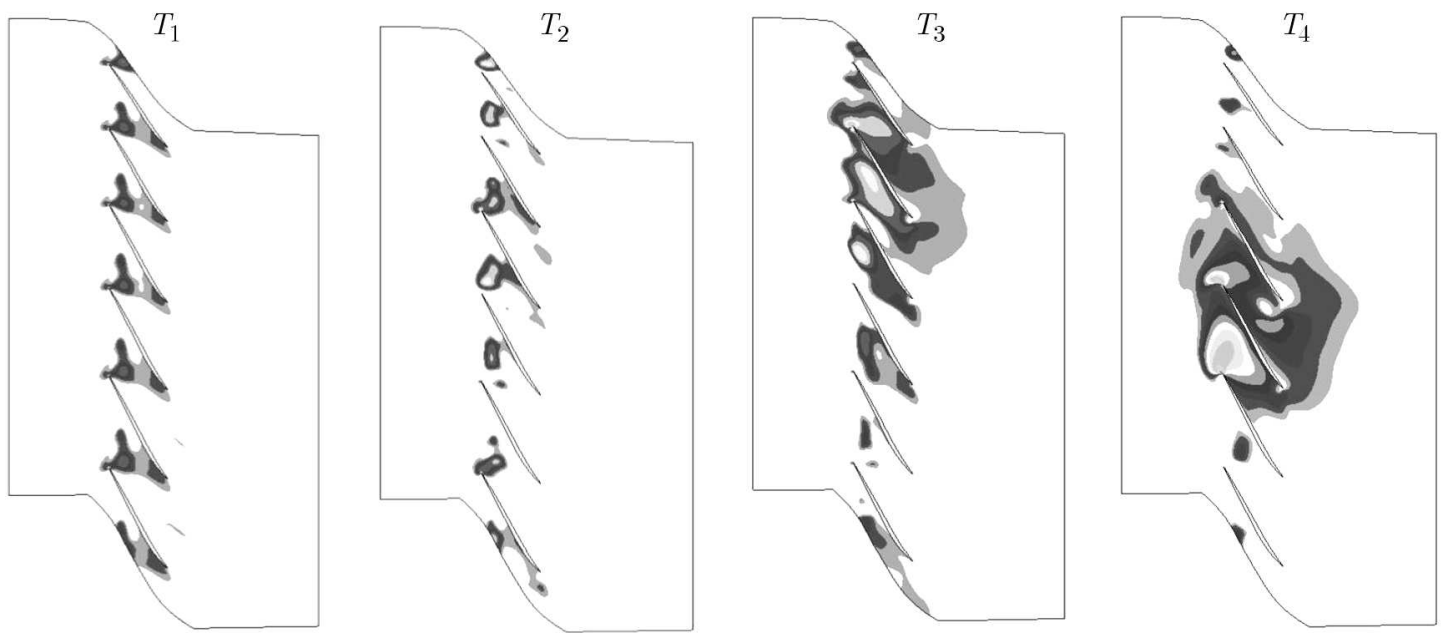

Fig. 6. The axial negative velocity distribution at the blade tip in 4 time instants
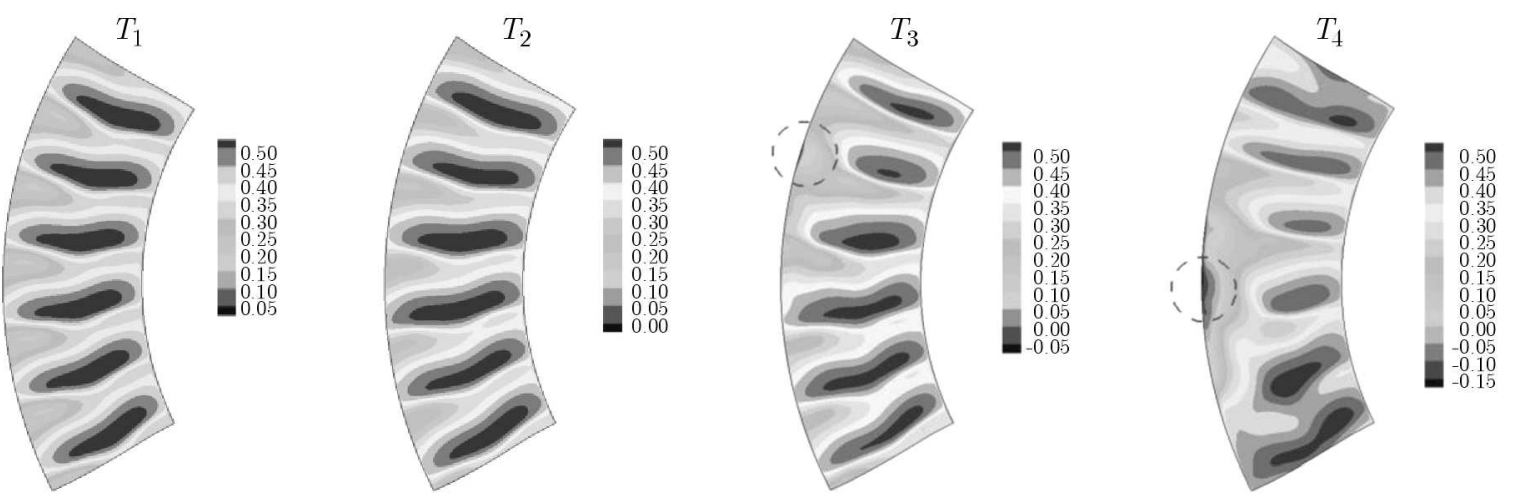

Fig. 7. The axial negative velocity distribution on $1 / 3$ of the axial chord plane in 4 time instants

\subsection{Discussion of stall inception mechanism}

The above analysis shows that the flow in the tip area has a certain correlation with the stall inception. For clarifying the flow physics of rotating stall inception, Hoying et al. (1999) developed a criterion for stall inception when the trajectory of the tip clearance vortex becomes perpendicular to the axial direction by using vortex kinematics arguments. Vo et al. (2008) demonstrated that stall inception is most likely accompanied by the forward spillage of tip clearance flow. Both of the two researches considered that tip clearance flow may contribute to the stall inception. In the transonic axial compressor, the interaction between the tip clearance flow and shock has been cited as one of the initiators of stall inception.

Here, the variety of the tip area flow from the stable condition to the near stall is illustrated clearly in order to discuss the flow physics of stall inception. Figure 8 shows the comparison of pressure and Mach number distributions at the blade tip. Figure 9 shows the static pressure coefficient distribution at the suction surface. An attached oblique passage shock, which is colored by the carnation dashed line, originates from the leading edge of the blade and extends across the passage to the suction surface of the adjacent blade at the stable condition shown in Fig. 8a. However, the shock detaches from the leading edge at the near stall condition (as shown in Fig. 8b). Two green vertical dashed lines in Fig. 9 present the position change of the static pressure coefficient sudden jumps, which indicates that the shock area at the suction surface shifts forward from $56 \%$ of the chord at the stable condition to $42 \%$ of the chord at the near stall condition. Additionally, see Fig. 8, the tip clearance vortex and shock intensity are higher at the near stall condition compared with the stable condition. Therefore, the interaction between the 
tip clearance flow and shock shifts forward, which is so intense that can induce the vortex to breakdown, and to cause a large blocked low momentum area downstream the passage shock at the near stall.
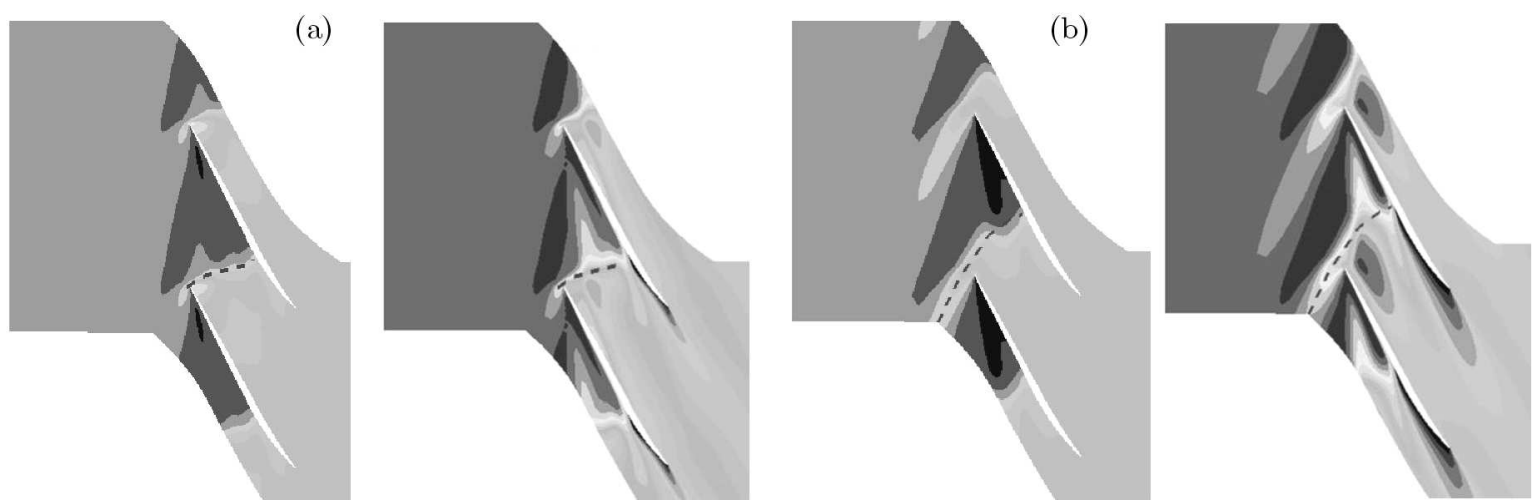

Fig. 8. Pressure and Mach number distributions at the blade tip: (a) stable condition and (b) near stall

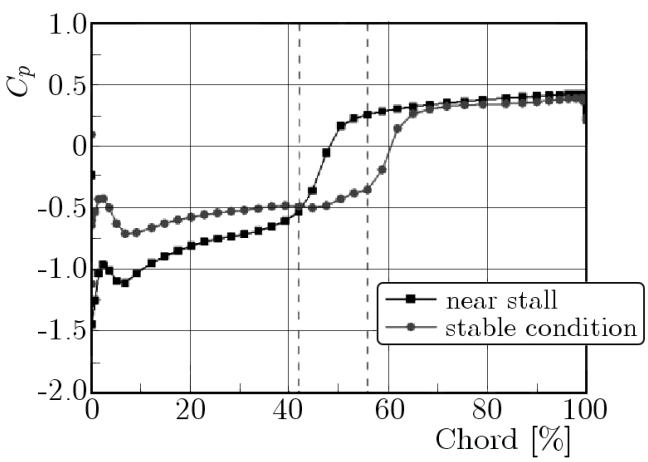

Fig. 9. The static pressure coefficient distribution at the suction surface of the blade tip at the stable condition and near stall
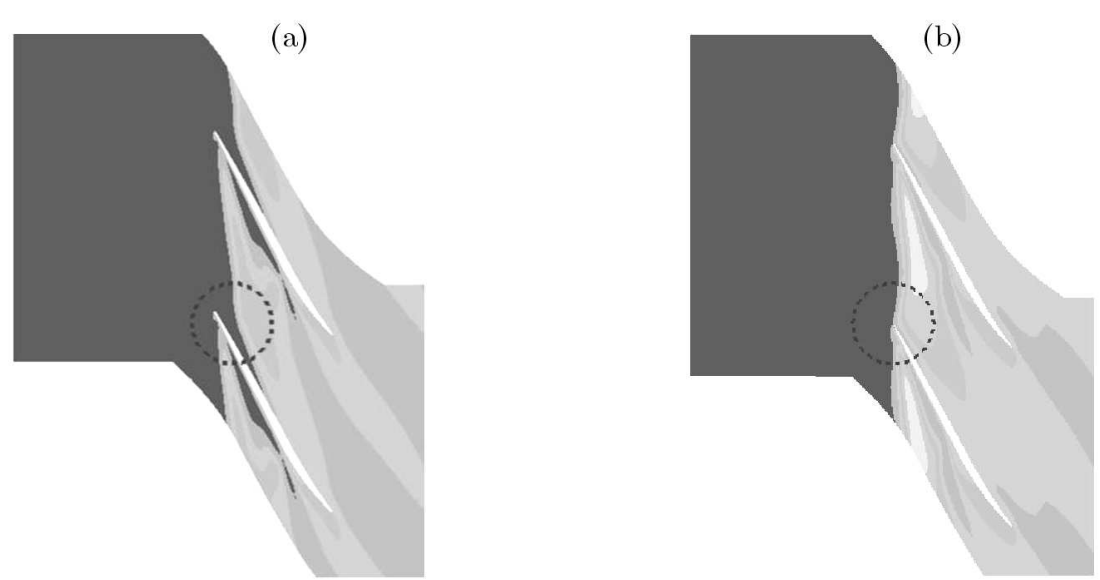

Fig. 10. Entropy distributions at the blade tip: (a) stable condition and (b) near stall

From many relevant reports, the distribution of relative total pressure or entropy can indicate the loss and interface between the incoming flow and tip clearance flow. Figure 10 shows the entropy distributions at the blade tip at the stable and near stall conditions. From these figures, we can see the interface at the near stall condition is closer to the leading edge than the stable condition. With a decrease in the mass flow rate, the incoming flow momentum intensity becomes lower. Thus, the more intense interaction between the shock and tip clearance vortex lead to large blockage, and shift the interface forwards. 
The clearance flow streamlines for both stable and near stall conditions are shown in Fig. 11. At the stable condition, the tip clearance vortex originates form the tip leading edge. The trajectory of the tip clearance vortex is not perpendicular to the axial direction. The oblique shock intersecting the vortex only slightly alters the shape of the vortex. In the case of near stall condition, the tip clearance vortex still originates form the tip leading edge. Some portion of the vortex trajectory becomes perpendicular to the axial direction. This trend follows with the stall inception criterion of Hoying et al. (1999). However, the shape of the clearance flow distorts, and its trajectory is pushed backward by the high pressure near the leading edge. The clearance flow does not spill over into the adjacent blade passage at the near stall condition. Figures 9 and 12 depict the shock and interface transition numerically. From the two figures, we can clearly see that the shock and tip clearance flow move upstream as the stall condition comes.
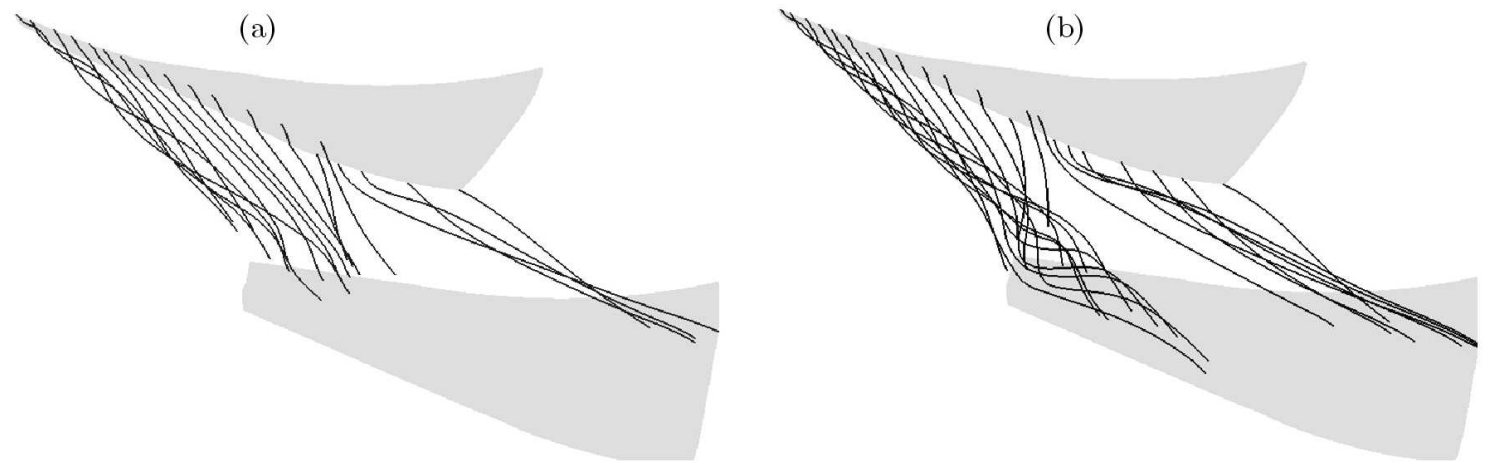

Fig. 11. Streamlines of the clearance flow: (a) stable condition and (b) near stall

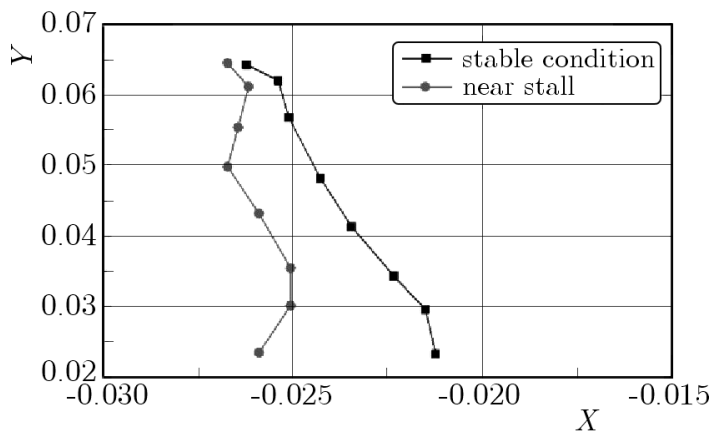

Fig. 12. Interface locations at the stable condition and stall conditions

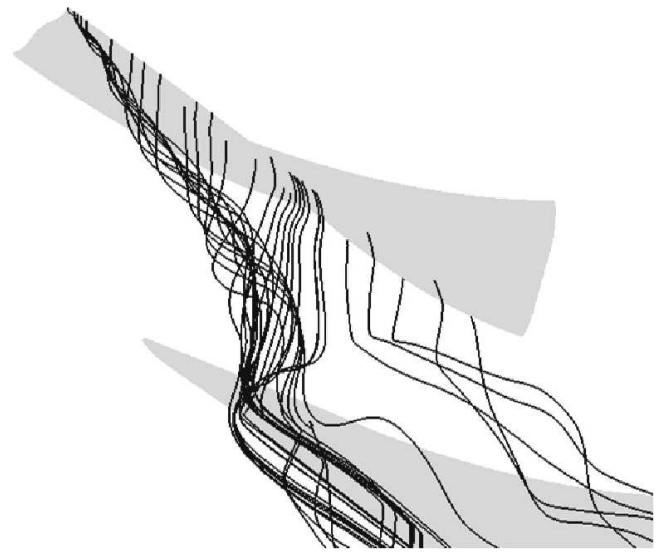

Fig. 13. Flow streamlines at the tip region at time $T_{2}$

Figure 13 shows flow streamlines at time $T_{2}$ in the stall inception simulation. The tip clearance flow shifts forward to the leading edge of the blade and spills over into the adjacent blade passage. 
The current numerical simulation has demonstrated that the spillage of the tip clearance flow near the blade leading edge and detached shock are the remarkable mechanism of rotating stall inception in the current transonic compressor rotor.

\section{Conclusion}

The stall inception flow fields in the transonic axial compressor rotor have been investigated by unsteady RANS simulations using the throttle model. Through the comparison between flows in the tip area at different conditions, the results can be summarized as follows:

- The stall inception process is simulated by the throttle model sufficiently. The stall starts from the tip area, and only one stall cell exists. Then the stall cell extends in the axial, circumferential and radial directions as the blade rotates.

- The interaction between the tip clearance vortex and the shock becomes stronger as the flow rate decreases. At the near stall condition, the low momentum fluid exists downstream the shock wave in the rotor passage, which brings larger blockage effects than at the stable condition.

- Under the effects of the large blockage of shock, tip clearance vortex and lower inflow momentum, the interface between the incoming flow and the tip clearance flow shifts forward. Finally, the clearance flow spills into the adjacent blade passages while the shock detaches from the leading edge, which contributes to the rotor stall process.

\section{References}

1. Chen J.-P., Hathaway M.D., Herrick G.P., 2008, Prestall behavior of a transonic axial compressor stage via time-accurate numerical simulation, Journal of Turbomachinery, 130, 4, 041014

2. Cumpsty N.A., 1989, Compressor Aerodynamics, Longman Scientific \& Technical, London

3. Davis R.L., YAO J., 2006, Axial compressor rotor flow structure at stall-inception, 44th AIAA Aerospace Sciences Meeting and Exhibition, 9-12 January, Reno, Nevada

4. DAY I., 1993, Stall inception in axial flow compressors, Journal of Turbomachinery, 115, 1, 1-9

5. Emmons H.W., Pearson C.E., Grant H.P., 1955, Compressor surge and stall propagation, Journal of Turbomachinery, Transactions of the ASME, 77, 455-467

6. Furukawa M., Saiki K., Yamada K., Inoue M., 2000, Unsteady flow behavior due to breakdown of tip vortex in an axial compressor rotor at near-stall condition, ASME-TURBO-EXPO, Munich, Germany

7. Hah C., Bergner J.R., Schiffer H.-P., 2006, Short length-scale rotating stall inception in a transonic axial compressor: criteria and mechanisms, ASME Turbo Expo 2006: Power for Land, Sea, and Air, Barcelona, Spain

8. Hah C., Rabe D.C., Wadia A.R., 2004, Role of tip-leakage vortices and passage shock in stall inception in a swept transonic compressor rotor, ASME Turbo Expo 2004: Power for Land, Sea, and Air, Vienna, Austria

9. Hah C., Schulze R., Wagner S., Hennecke D.K., 1999, Numerical and experimental study for short wavelength stall inception in a low-speed axial compressor, Proceedings of 14 th International Symposium Air Breathing Engines, Florence, Italy

10. He L., 1997, Computational study of rotating-stall inception in axial compressors, Journal of Propulsion and Power, 13, 1, 31-38

11. Hoying D.A., Tan C.S., Greitzer E., Vo H.D., 1999, Role of blade passage flow structures in axial compressor rotating stall inception, Journal of Turbomachinery, 121, 4, 735-742 
12. Hu J.F., Zhu X.C., Ou-Yang H., Tian J., Wu Y.D., Qiang X.Q., Zhao G., Du Z.H., 2013, The unsteadiness of tip clearance flow and its effect to stability of transonic axial compressor, Journal of Theoretical and Applied Mechanics, 51, 2, 431-438

13. Moore F., Greitzer E., 1986, A theory of post-stall transients in axial compression systems: Part I - Development of equations, Journal of Engineering for Gas Turbines and Power, 108, 1, 68-76

14. NiAzI S., 2000, Numerical simulation of rotating stall and surge alleviation in axial compressors, Ph.D. Thesis, Georgia Institute of Technology

15. Vo H.D., Tan C.S., Greitzer E.M., 2008, Criteria for spike initiated rotating stall, Journal of Turbomachinery, 130, 1, 011023

16. Yamada K., Funazaki K., Furukawa M., 2007, The behavior of tip clearance flow at near-stall condition in a transonic axial compressor rotor, ASME Turbo Expo 200\%: Power for Land, Sea, and Air, Montreal, Canada

17. Yao J., Cargill P.L., Holmes D.G., Gorrell S.E., 2010, Aspects of numerical analysis for unsteady flows in aircraft engines, 48th AIAA Aerospace Sciences Meeting Including the New Horizons Forum and Aerospace Exposition, Orlando, Florida

Manuscript received April 16, 2014; accepted for print September 5, 2014 